\title{
DONNÉES SUR LA CROISSANCE DE L'ANGUILLE (ANGUILLA ANGUILLA L.) DANS LE COURS MOYEN DU RHIN, RÉGION ALSACIENNE.
}

\author{
F.J. MEUNIER (1) (2)
}

(1) Laboratoire d'Ichtyologie générale et appliquée, Muséum National d'Histoire Naturelle, 43 rue Cuvier, 75231 PARIS Cedex 05, France.

(2) URA CNRS 1137, Formations Squelettiques, Université D. Diderot, Case 7077, 2 Place Jussieu, 75251 PARIS Cedex 05, France.

\section{RÉSUMÉ}

La capture régulière, pendant cinq années (1990-94), d'anguilles marquées à la tétracycline au stade civelle et déversées dans le Rhin en mars 1989, confirme la forte variabilité individuelle de leur croissance. La croissance moyenne annuelle, sexes confondus, est de $55 \mathrm{~mm}$, avec un accroissement voisin de $100 \mathrm{~mm}$ lors de la première année de vie en eau douce. Ces valeurs se situent dans les limites des résultats obtenus pour d'autres rivières, lacs ou lagunes de l'Europe occidentale.

Mots-clés : croissance, Anguilla anguilla L., milieu naturel, marquage vital.

\section{GROWTH DATA OF EEL (ANGUILLA ANGUILLA L.) \\ IN THE ALSACIAN PART OF THE RIVER RHINE.}

\section{SUMMARY}

Elvers labelled with tetracycline were put in the river Rhine during March 1989 and regularly sampled over a five years period. The catches confirmed high individual variability of their growth. The mean annual growth rate of unsexed fish is around $55 \mathrm{~mm}$ with an increment near $100 \mathrm{~mm}$ for the first year of life in freshwater. These data fall between the two extremes obtained in other rivers, lakes and lagoons of western Europe.

Key-words : growth, Anguilla anguilla L., vital labelling.

\section{INTRODUCTION}

Comme l'a signalé récemment FONTAINE (1992) et contrairement aux affirmations de BERTIN à la lecture des travaux de Schmidt (BERTIN, 1942), bien des aspects de la biologie de l'anguille, espèce commune de nos rivières, sont longtemps restés obscurs. A l'heure actuelle encore, même si des progrès incontestables de nos connaissances ont été réalisés aux cours des deux dernières décennies (TESCH, 1977 ; BRUSLÉ, 1989 ; FONTAINE, 1989 ; LECOMTE-FINIGER et YAHYAOUI, 1989 ; LECOMTE-FINIGER, 1990, 1992 ; TZENG et TSAI, 1992, 1994 ; ...), des domaines comme celui de la croissance restent l'objet de controverses. Les connaissances sur la croissance de l'anguille reposent souvent sur des observations relativement anciennes peut-être sujettes à discussion. De plus, ce modèle biologique, grand migrateur, est d'une approche difficilẹ et il se prête mal à l'expérimentation.

Les études de croissance et/ou de dynamique de population, comme pour de très nombreuses espèces de téléostéens, nécessitent de répartir les échantillonnages en classes d'âge bien différenciées. Or, pour ce travail avec l'anguille, on ne dispose, en 
dehors de la méthode de Petersen qui donne des classes d'âge moyen (FONTENELLE, 1991), que de l'otolithométrie comme technique d'estimation de l'âge individuel, technique particulièrement délicate à mettre en oeuvre chez cette espèce (DEELDER, 1981-82 ; LECOMTE-FINIGER, 1985 ; MICHAUD et al., 1988 ; VØLLESTAD et al., 1988) ; les écailles sont, elles, inutilisables (DEELDER, 1970 ; RUIZHANG GUAN et al., 1994). L'ensemble de ces difficultés explique, probablement en partie, pourquoi les résultats de la littérature scientifique, sur les potentialités de croissance de cette espèce dans son milieu naturel, apparaissent plutôt disparates (Fig. 1) et nous manquons d'études basées sur une validation absolue de l'âge des animaux. Mais, comme nous le verrons plus loin, la plupart des travaux récents mettent l'accent, comme l'avaient déjà mentionné les anciens auteurs (BERTIN, 1942), sur une particularité de l'anguille européenne, sa forte variabilité individuelle (BERG, 1990). Celle-ci est très nette entre divers habitats (VØLLESTAD, 1989, 1992 ; FONTENELLE, 1991) ou à l'intérieur d'une population qu'elle soit d'estuaire (FERNÀNDEZ-DELGADO et al., 1989), de rivière (MORIARTY, 1983 ; VØLLESTAD et JONSSON, 1988 ; POOLE, 1991) ou de lac (PAULOVITS et BIRÕ, 1986 ; NAGIEC et BAHNSAWY, 1990). Ces résultats sont confirmés chez $A$. anguilla dans les fermes d'élevage (EGUSA, 1979 ; KUHLMANN, 1979 ; WICKINS, 1985) et chez les espèces voisines dans leur milieu naturel : $A$. rostrata (HANSEN et EVERSOLE, 1984), A. mossambica (McEWAN et HECHT, 1984) et $A$. australis (CHISNALL et HAYES, 1991). Cette variabilité individuelle, pour ce qui concerne la croissance, est très probablement sous l'influence de divers facteurs: âge, sexe, saisonalité, particularités du milieu,... pour ne pas parler des aspects génétiques (PANFILI et al., 1994). Par ailleurs, dans le cas d'études expérimentales, les manipulations effectuées sur les animaux, comme le marquage individuel (NIELSEN, 1988), créent un stress qui peut fausser les résultats en provoquant jusqu'à $50 \%$ de réduction de la croissance (BERG, 1986).

Des progrès sensibles dans la précision des données acquises sur la croissance ont été effectués cette dernière décennie grâce aux développements récents de la technologie. Une première série d'améliorations est due à l'utilisation du microscope électronique à balayage pour la description et l'analyse des différentes marques de croissance des otolithes et, en conséquence, pour leur interprétation critique (MOUNAIX, 1992 ; PANFILI, 1993). Ces auteurs ont ainsi montré que la nature des stries des otolithes pouvait refléter de façon précise les qualités du milieu où vivent les animaux. Par exemple, compte tenu de la régularité des marques de croissance annuelles sur les otolithes, les spécimens de l'estuaire de la Vilaine semblent bénéficier de meilleures conditions de vie que dans les secteurs plus amont de la rivière où les marques forment des ensembles plus complexes et leur vitesse de croissance y est plus rapide (MOUNAIX, 1992). Une deuxième série de progrès est l'utilisation du marquage vital, après les travaux de DEKKER (1986), comme moyen de validation des estimations d'âge et qui permet de préciser, pour chaque spécimen marqué, la croissance absolue pendant la période expérimentale (MOUNAIX, 1992 ; PANFILI, 1993). Dans ces deux études, les observations n'ont pas dépassé 18 mois mais elles renforcent, toutefois, sérieusement l'hypothèse d'une variabilité individuelle de la croissance chez l'anguille.

Des résultats récents acquis au cours d'une expérience d'alevinage en milieu naturel d'animaux marqués à la tétracycline au stade civelle apportent des données intéressantes sur une période beaucoup plus longue, puisque le travail expérimental (toujours en cours) s'est déjà développé sur 5 années.

\section{MATÉRIEL ET MÉTHODES}

Dans le cadre d'une étude de la biologie de l'anguille Anguilla anguilla dans le Rhin (Fond Sandoz pour le Rhin) effectuée entre Bâle et Mannheim (pour plus de détails, voir MEUNIER, 1994) et concernant : a) l'importance des populations ; b) le recrutement naturel et/ou repeuplement ; c) la dynamique de population ; d) la vitesse de croissance, nous avons pu obtenir des données précises sur la croissance in situ à partir d'un alevinage de $500 \mathrm{~kg}$ de civelles (șoit près de 1250000 individus). Les civelles ayant été au préalable marquées chimiquement, la croissance des animaux a été suivie sur cinq années. L'avantage de cette méthode de marquage est d'obtenir une validation absolue de l'âge de l'animal en expérimentation in situ (BEAMISH et McFARLANE, 1983). 


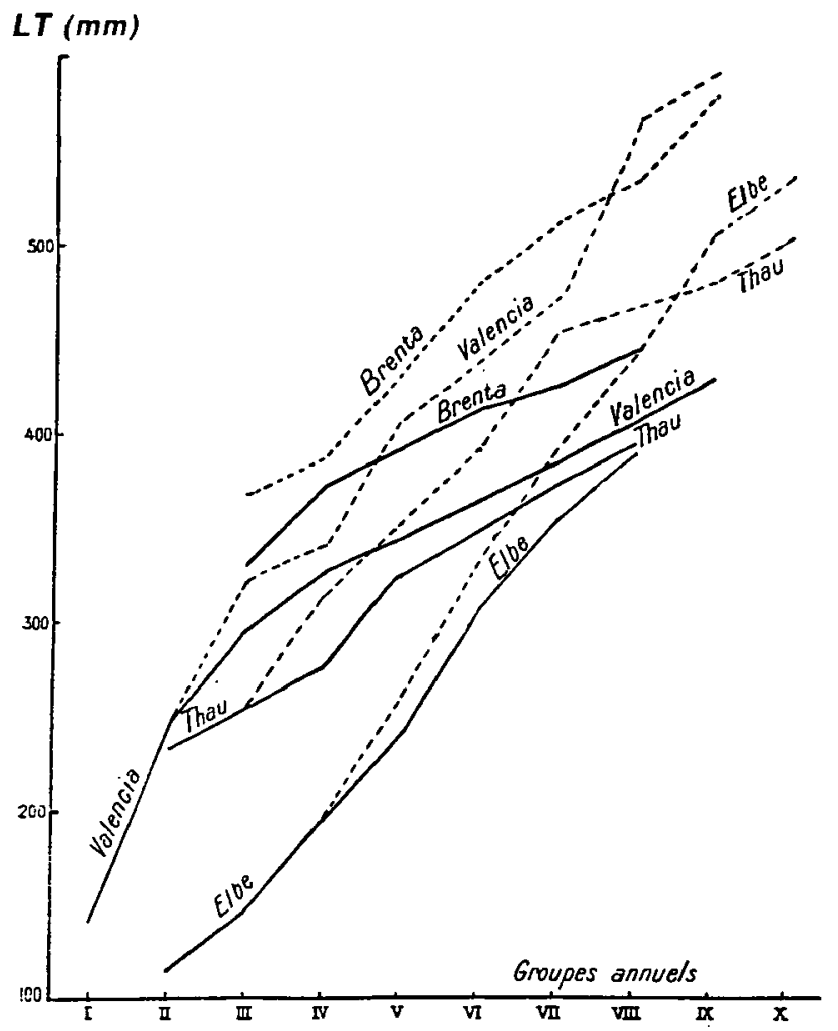

Figure 1 : Courbes de croissance de l'anguille dans différents fleuves et lagunes d'Europe. En trait continu les animaux immatures et les mâles, en pointillé les femelles (d'après BERTIN, 1942). La Brenta est une rivière d'Italie au sud-ouest de Venise et "Valencia" correspond à la lagune Albufera située juste au sud de la ville espagnole.

Flgure 1 : Growth of eel in various European rivers and lagoons. Straight line males, dotted line females (after BERTIN, 1942). The Brenta is a river of Italia in the south-west of Venizia and "Valencia» correspond to the lagoon Albufera in the south of the Spanish town.

Les animaux ont été marqués, en Mars 1989, avec du chlorhydrate de tétracycline par balnéation massive (technique de balnéation-choc osmotique ; ALCOBENDAS et al., $1991,1992)$, les organes cibles étant les otolithes. Les civelles, au moment du marquage, mesuraient (LT) de 61 à $76 \mathrm{~mm}(\mathrm{M}=70 \mathrm{~mm})$ et avaient un poids moyen de $256 \mathrm{mg}$; elles ont été déversées dans les secteurs d'alevinage moins de $48 \mathrm{~h}$ après la balnéation (Fig. 2 ; pour plus de détails voir MEUNIER, 1994).

Des échantillons annuels de contrôle ont été capturés régulièrement à Kembs, Rhinau et Gambsheim, au printemps et au début de l'automne de 1990 à 1994, soit 5 années de suivi (Fig. 2). Un total de 116 anguilles, issues de l'alevinage de Mars 1989 et possédant des otolithes marqués à la tétracycline, a été capturé au long de ces cinq années. Les otolithes prélevés sont nettoyés, séchés et observés avec un microscope à fluorescence ; les observations sont faites à sec pour les plus petits otolithes (anguilles de 1 à 3 ans), dans l'alcool $70^{\circ}$ pour les plus gros (anguilles de 4 et 5 ans). Le nombre d'annuli est ensuite compté pour s'assurer de l'appartenance de chaque animal au stock d'alevinage expérimental. 


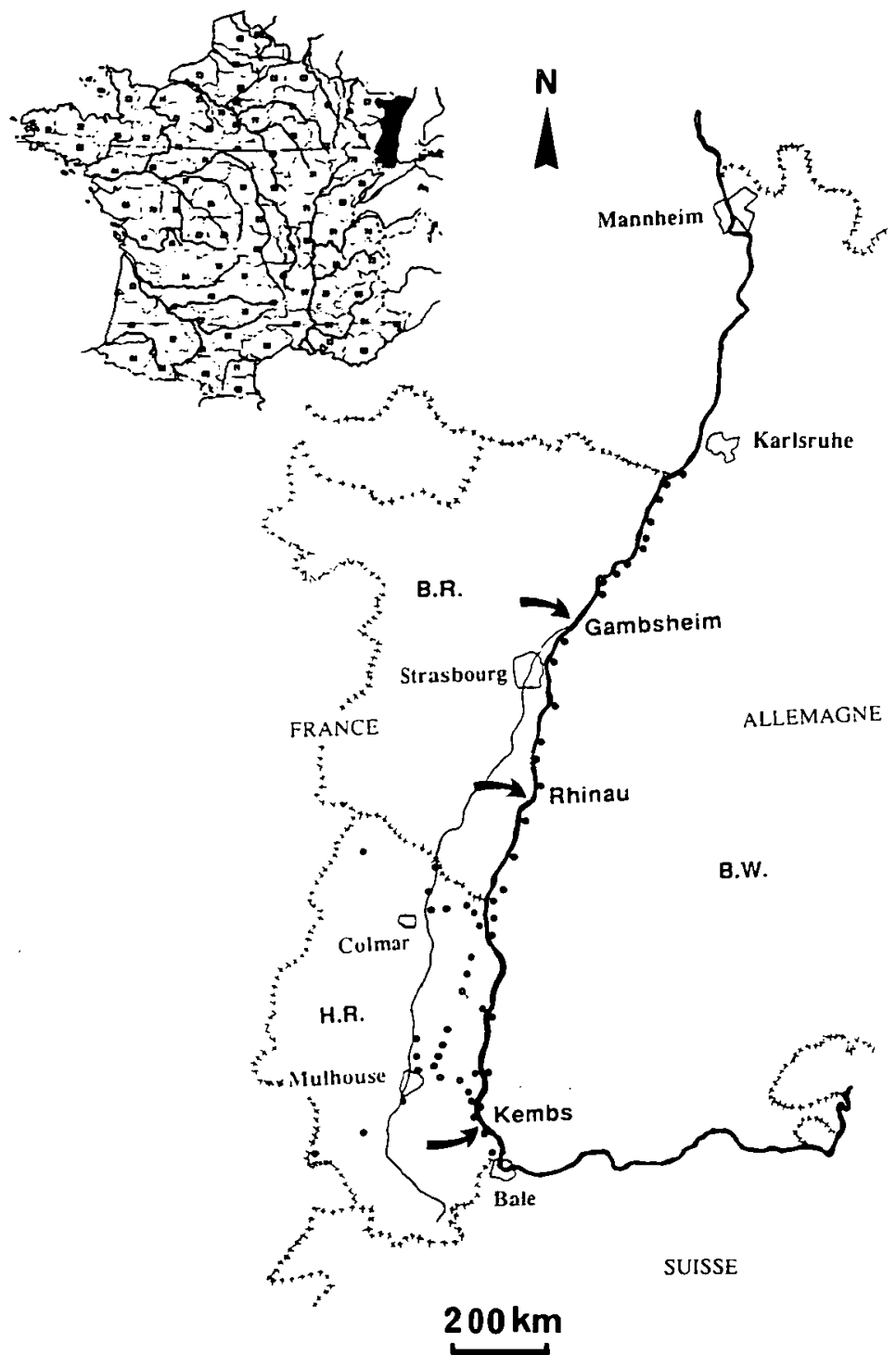

Flgure 2 : Carte des alevinages (points noirs) et des sites d'échantillonnages (flèches) dans les départements du Bas-Rhin (B.R.) et du Haut-Rhin (H.R.) (d'après MEUNIER, 1994).

Flgure 2 : Map showing the elver stocking (black points) and the localization of sampling.(arrows) in the department of «Bas-Rhin» (B.R.) and «Haut-Rhin» (H.R.) (after MEUNIER, 1994).

\section{RÉSULTATS-DISCUSSION}

Les marques jaune d'or de la tétracycline sont observées sans difficultés majeures sur des otolithes entiers, même pour les animaux de 5 ans. Elles apparaissent d'autant plus éloignées du bord de l'otolithe que les animaux recapturés sont plus vieux (Fig. 3). La méthode de marquage des civelles par balnéation (ALCOBENDAS et al., 1991, 1992) est la seule qui permette de préparer un très grand nombre de spécimens et s'avère supérieure à toutes les autres (NIELSEN, 1988). Chaque résultat individuel est une valeur de croissance absolue validée par la présence de la tétracycline. Ce n'est pas rigoureusement le cas à partir d'une estimation d'âge par otolithométrie qui peut sousévaluer ou surévaluer l'âge selon la méthode d'obsenation (VØLLESTAD, 1985). 
Bull. Fr. Pêche Piscic. (1994) 335 : 133-144 - 137 -

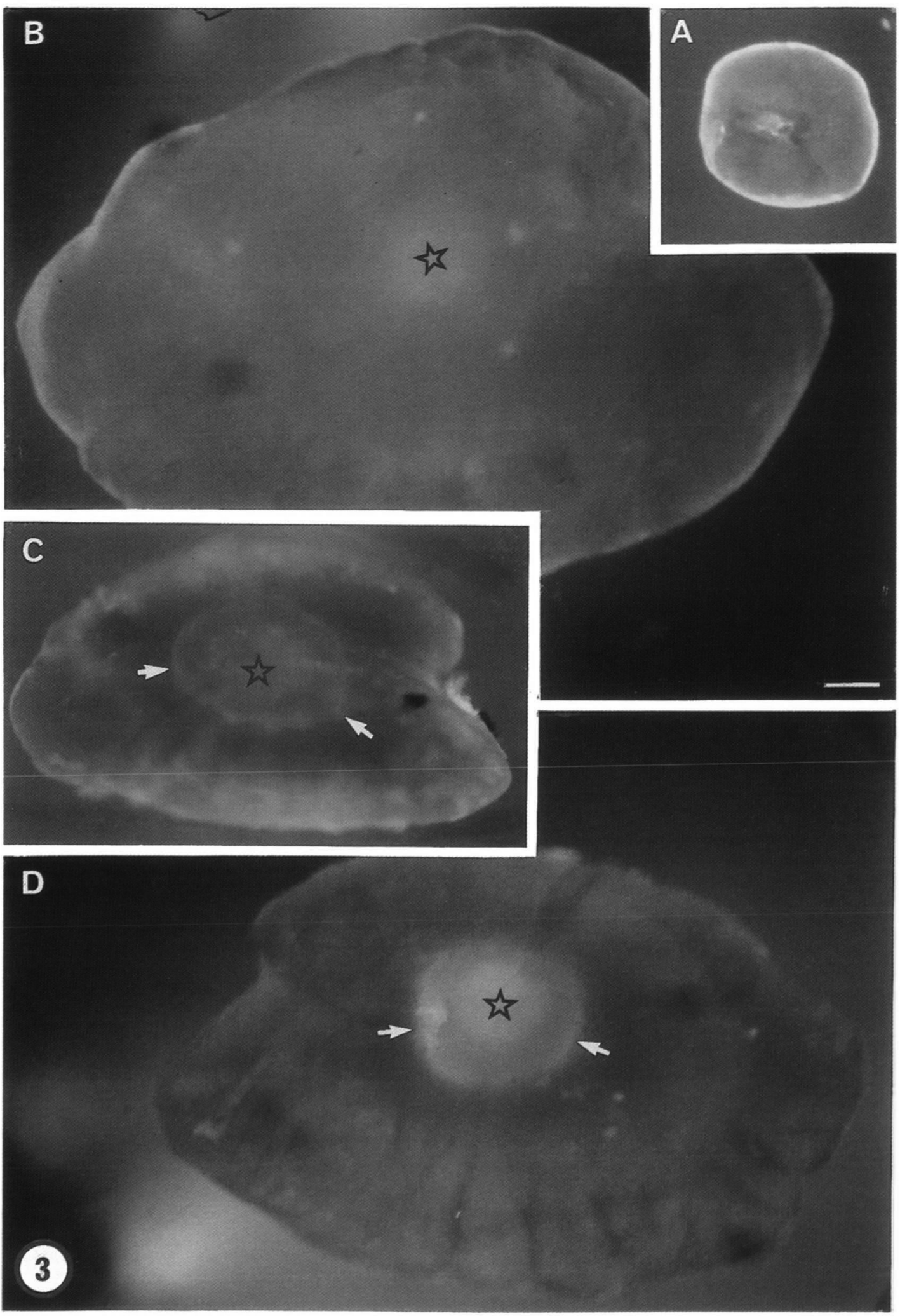

Figure 3 
Figure 3 : A) Otolithe d'une civelle marqué à la tétracycline et prélevé 15 jours après la balnéation : la marque fluorescente est marginale.

B) Otolithe témoin d'une anguille non marquée de la classe $3^{+}$(capturée à Kembs en 1992) : pas d'anneau jaune visible, seul le nucleus (étoile) émet une légère fluorescence.

C) Otolithe positif d'une anguille de la classe $1^{+}$(capturée à Rhinau en 1990).

D) Otolithe positif d'une anguille de la classe $3^{+}$(capturée à Kembs en 1992). On remarque sur les deux images $C$ et $D$ que le diamètre de la marque fluorescente est identique à celui de la civelle témoin de la figure A. (Echelle $=100 \mu \mathrm{m}$ ).

FIgure 3 : A) Otolith of an elver labelled with tetracycline and sampled 15 days after balneation : the fluorescent line is at the margin.

B) Control otolith of an unlabelled eel of class $3^{+}$(from Kembs, 1992) : there is no yellow mark, the nucleus only (star) shows a tiny fluorescence.

C) Labelled otolith from an eel of class $1^{+}$(from Rhinau, 1990). 
La taille des anguilles échantillonnées possédant des otolithes marqués à la tétracycline est reportée en fonction du temps sur la figure 4. On constate d'emblée une importante dispersion des points dans chaque échantillon de prélèvement. Ceci traduit la variabilité individuelle de la croissance en longueur des animaux vivant dans le Rhin. Une forte croissance est observée pendant la première année avec un gain moyen de près de $100 \mathrm{~mm}$. L'accroissement moyen annuel (calculé sur les échantillons de printemps) sur les cinq années de suivi est de $55 \mathrm{~mm}$ avec des valeurs comprises entre 26 et $98 \mathrm{~mm} / \mathrm{an}$. Aucune distinction des sexes n'ayant été faite sur ces différents échantillons, il ne nous est pas possible de comparer les performances relatives de croissance entre mâles et femelles. Ces dernières grandissent en général plus vite que les mâles et atteignent des tailles très supérieures ayant par ailleurs une plus grande longévité (BERTIN, 1942 ; DEELDER, 1970 ; ROSSI et VILLANI, 1980 ; VØLLESTAD et JONSSON, 1988 ; PANFILI et al., 1994).

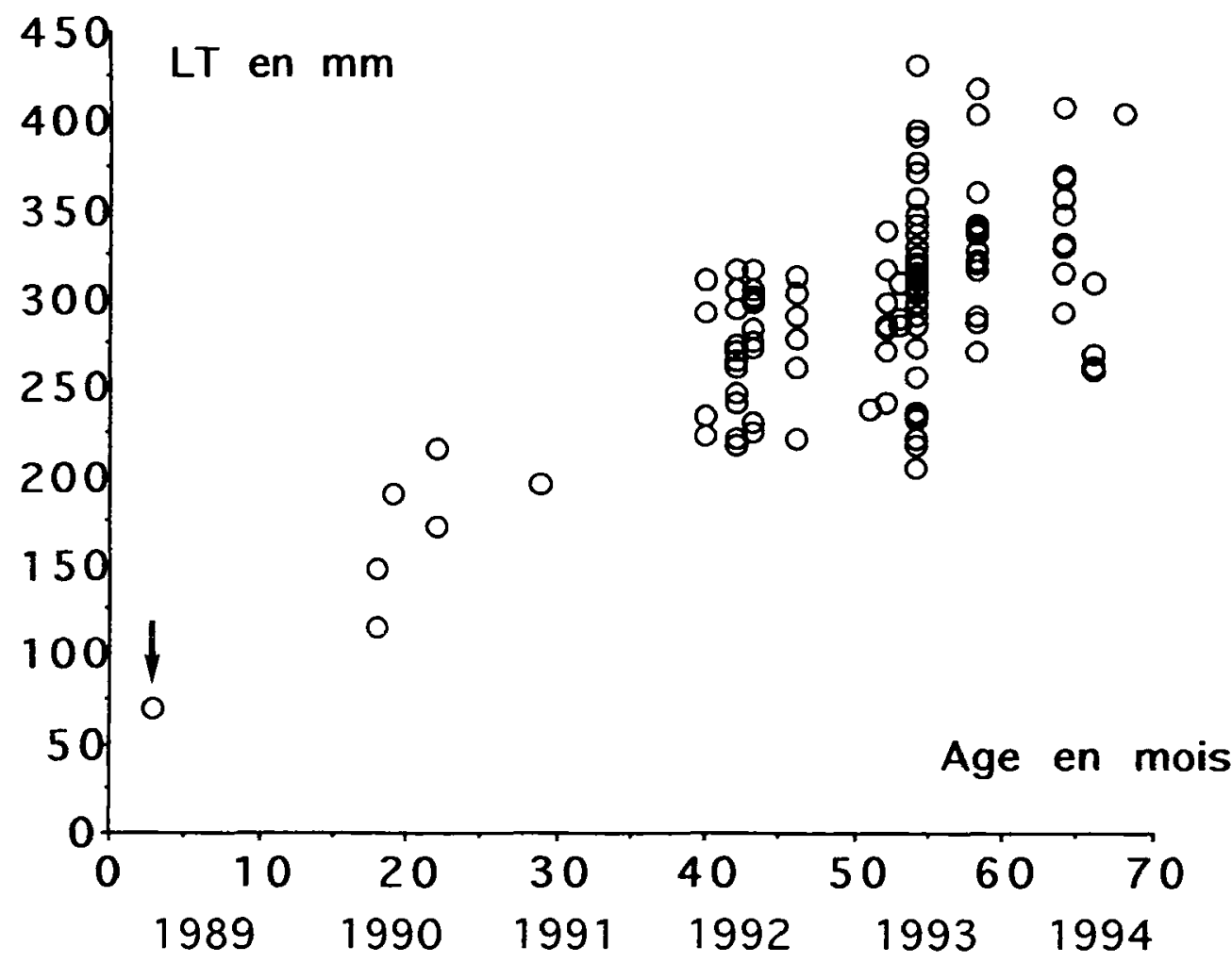

Figure 4 : Longueur totale (en $\mathrm{mm}$ ) des anguilles expérimentales marquées à la tétracycline en fonction du temps (en mois) à partir de Janvier 1989 (flèche = date de l'alevinage, Mars 1989).

Figure 4 : Total length (in $\mathrm{mm}$ ) of experimental eels labelled with tetracycline against time (in month) since January 1989 (arrow = elvers stocking, March 1989).

A notre connaissance il existe peu ou pas de données, dans la littérature spécialisée, sur la croissance de l'anguille dans le Rhin. Seuls sont disponibles des résultats sur la croissance des mâles dans l'IJsselmeer que nous pouvons considérer comme faisant partie d'un même grand bassin versant (Tab. I ; DEELDER, 1957). L'accroissement moyen annuel y serait de 40 à $50 \mathrm{~mm}$ avec une fourchette s'étalant de 20 à $80 \mathrm{~mm} / \mathrm{an}$. Ce taux moyen de croissance annuelle est donc proche de celui du Rhin alsacien puisque nos résultats donnent une valeur de $55 \mathrm{~mm} / \mathrm{an}$, même si nous n'avons 
pas séparé mâles et femelles. En revanche, le gain de la première année est nettement plus faible dans l'IJsselmeer où on peut l'estimer à $40-50 \mathrm{~mm}$ (Tab. I). La croissance des anguilles dans le Rhin alsacien lors de la première année est nettement supérieure à celles de la Vilaine, mais très rapidement, on observe un rééquilibrage tout au moins avec celles de l'estuaire (Tab. I). En revanche, les performances des anguilles expérimentales alsaciennes sont inférieures à celles du Bassin d'Arcachon où les tailles respectives atteintes lors des trois premières années sont 201 (soit un gain d'environ $130 \mathrm{~mm}$ la 1ère année), 271 et $342 \mathrm{~mm}$ (LEE et LASSERRE, 1979). En Camargue également, l'accroissement annuel moyen des anguilles lors des 2ème, 3ème et 4ème années est de 50 à $75 \mathrm{~mm} / \mathrm{an}$ (PANFILI et al., 1994). Au vu de l'ensemble de ces résultats sur différents systèmes aquatiques français, il semble donc que les facteurs physiques et biologiques du Rhin moyen soient relativement favorables à la croissance de l'anguille.

Tableau I : Tailles annuelles moyennes (en $\mathrm{mm}$ ) atteintes par les anguilles dans différents milieux sur une période de 10 ans (valeurs observées ou " " rétrocalculées). 1 : VØLLESTAD, 1986 ; 2 : NAGIEC et BAHNSAWY, 1990 ; 3 : RASMUSSEN et THERKILDSEN, $1979 ; 4:$ SINHA et JONES, $1967 ; 5$ : MOUNAIX, 1992 ; 6 : FERNÀNDEZ-DELGADO et al., $1989 ; 7$ : GORDO et JORGE, 1991 ; 8 : DEELDER, 1957 ; 9 : présent travail.

Table I : Mean yearly length (in $\mathrm{mm}$ ) of the eels in various freshwater areas during the first 10 years of life (observed data or " " back-calculated data). 1 : VØLLESTAD, 1986 ; 2 : NAGIEC and BAHNSAWY, 1990 ; 3 : RASMUSSEN and THERKILDSEN, $1979 ; 4$ : SINHA and JONES, $1967 ; 5$ : MOUNAIX, 1992 ; 6 : FERNÀNDEZ-DELGADO et al., $1989 ; 7$ : GORDO and JORGE, $1991 ; 8$ : DEELDER, 1957 ; 9 : this study.

\begin{tabular}{|c||c|c|c|c|c|c|c|c||c|c|}
\hline \multirow{2}{*}{ Age } & $\begin{array}{c}1 \\
\text { fjord } \\
\text { Norvège }\end{array}$ & $\begin{array}{c}2 \\
\text { lac } \\
\text { Pologne }\end{array}$ & $\begin{array}{c}3 \\
\text { rivière } \\
\text { Danemark }\end{array}$ & $\begin{array}{c}4 \\
\text { rivière } \\
\text { Angleterre }\end{array}$ & $\begin{array}{c}\text { estuaire } \\
\text { Vilaine }\end{array}$ & $\begin{array}{c}6 \\
\text { rivière } \\
\text { Vilaine }\end{array}$ & $\begin{array}{c}7 \\
\text { estuaire } \\
\text { Espagne }\end{array}$ & $\begin{array}{c}8 \\
\text { lagune } \\
\text { Portugal }\end{array}$ & $\begin{array}{c}9 \\
\text { lagune } \\
\text { IJsselmeer }\end{array}$ & $\begin{array}{c}\text { Rhin } \\
\text { moyen }\end{array}$ \\
\hline 1 & 115 & $" 135^{\prime \prime}$ & 108 & 136 & 126 & 140 & $" 156 "$ & 172 & 110 & 168 \\
\hline 2 & 200 & $" 183^{\prime \prime}$ & 153 & 162 & 198 & 235 & 308 & 233 & 160 & 195 \\
\hline 3 & 235 & $" 222^{\prime \prime}$ & 187 & 201 & 243 & 296 & 390 & 295 & 200 & 281 \\
\hline 4 & 290 & $" 268 "$ & 265 & 235 & 302 & 306 & 450 & 351 & 250 & 307 \\
\hline 5 & 335 & $" 306 "$ & 278 & 276 & 464 & 335 & $" 504 "$ & 401 & 280 & 346 \\
\hline 6 & 375 & $" 346 "$ & 344 & 302 & 619 & 451 & 541 & 456 & 315 & \\
\hline 7 & 420 & $" 382 "$ & 389 & & & & & 512 & 340 & \\
\hline 8 & 450 & $" 418 "$ & 400 & & & & & 550 & 360 & \\
\hline 9 & 470 & $" 459 "$ & 407 & & & & & 588 & & \\
\hline 10 & 500 & $" 496 "$ & & & & & & & & \\
\hline
\end{tabular}

Les valeurs moyennes des tailles par classe d'âge des anguilles du Rhin ont été comparées à celles d'autres habitats d'Europe. On constate que les performances de croissance de l'anguille dans la portion alsacienne du Rhin sont, dans l'ensemble, supérieures à celles de rivières et/ou lacs plus nordiques de Norvège, du Danemark, de Pologne ou d'Angleterre (Tab. I). Elles sont au contraire inférieures à celles de pays situés plus au sud comme le Portugal et l'Espagne (Tab. I). Les fortes valeurs données pour la région de Cadix (ARIAS et DRAKE, 1985), notamment pour la première année (253 mm, soit un gain d'accroissement voisin de $180 \mathrm{~mm}$ ), sont toutefois contestées (GORDO et JORGE, 1991). Les différences de croissance observées entre ces différents milieux qui montrent un effet "latitude" (FERNÀNDEZ-DELGADO et al., 1989) peuvent avoir des origines variées dont la température moyenne de l'eau mais aussi les spectres alimentaires, les particularités de l'habitat,... D'une façon générale, l'accroissement de la 
première année est assez nettement supérieur à celui des années suivantes (Tab. I). L'absence de spécimens de cette classe d'âge dans les études de croissance in situ peut conduire les évaluations d'âge par rétrocalcul à minimiser cet accroissement de la première année. Ainsi, les tailles proposées par rétrocalcul $(127 \mathrm{~mm}$ pour les mâles, $156 \mathrm{~mm}$ pour les femelles) pour les anguilles d'un an dans l'estuaire du Guadalquivir (FERNÀNDEZDELGADO et al., 1989) sont probablement inférieures à la réalité.

\section{CONCLUSION}

Le suivi régulier de la croissance, dans la portion alsacienne du Rhin, d'anguilles préalablement marquées à la tétracycline au moment de l'alevinage, permet d'obtenir des résultats particulièrement précis. Ces résultats confirment la majorité des données acquises par différents auteurs, au cours des vingt dernières années, sur la base d'estimations de l'âge individuel des animaux, grâce à l'otolithométrie essentiellement. L'originalité du présent travail est d'avoir mis en oeuvre une validation absolue des valeurs obtenues grâce au marquage vital dès les premiers temps de la vie en eau douce des civelles. La relative facilité pour retrouver des animaux expérimentaux dans les échantillonnages annuels de contrôle laisse présager la possibilité de poursuivre cette étude encore quelque temps.

\section{REMERCIEMENTS}

Ce travail a été réalisé grâce à la collaboration de $M$. ALCOBENDAS, J. CASTANET, H. FRANCILLON-VIEILLOT et F. LECOMTE (URA CNRS 1137, Equipe "Formations squelettiques", Université D. Diderot, 2 place Jussieu, 75251 Paris Cedex 05) et P. BOHN, T. CLAUSS, P. MAIRE et G. PANON (Conseil Supérieur de la Pêche, Délégation régionale $N^{\circ} 3,18$ rue de Nomeny, 57158 Montigny-lès-Metz).

Les balnéations ont été faites grâce à l'obligeance de Monsieur MABIT dans les Etablissements BEAUR à Rezé (Loire-Atlantique) et la tétracycline a été fournie gracieusement par les Etablissements SANDOZ.

L'ensemble du travail a bénéficié d'une aide du Conseil Supérieur de la Pêche : contrat du 30 Juin 1988.

\section{BIBLIOGRAPHIE}

ALCOBENDAS M., LECOMTE F., FRANCILLON-VIEILLOT H., CASTANET J., MEUNIER F.J., MAIRE P., 1991. Technique de marquage en masse de civelles (Anguilla anguilla $\mathrm{L}$.) par balnéation rapide dans le fluorochrome. Application au marquage à la tétracycline de $500 \mathrm{~kg}$ de civelles. Bull. Fr. Pêche Pisc., 321, 43-54.

ALCOBENDAS M., LECOMTE F., FRANCILLON-VIEILLOT H., CASTANET J., MEUNIER F.J., 1992. Marquage vital en masse chez l'anguille Anguilla anguilla à l'aide d'une technique de balnéation rapide. In BAGLINIERE J.L., CASTANET J., CONAND F. et MEUNIER F.J., Tissus durs et Age individuel des Vertébrés, 93-102. Colloques et Séminaires, ORSTOM-INRA, Paris.

ARIAS A.M., DRAKE P., 1985. Estructura de la poblacion y régimen alimentario de Anguilla anguilla L., 1758 (Osteichthyes, Anguillidae), en los esteros de San Fernando (Cadiz). Invest. Pesq., 49, 475-491.

BEAMISH R.J., MACFARLANE G.A., 1983. The forgotten requirement for age validation in fisheries biology. Trans. Am. Fish. Soc., 112, 735-743.

BERG R., 1986. Field studies on eel (Anguilla anguilla) in Lake Constance: tagging effects causing retardation of growth. Vie Milieu, 36, 285-286.

BERG R., 1990. The growth of eels : a critical assessment of data from open waters. Int. Revue ges. Hydrobiol., 75, 755-762. 
BERTIN L., 1942. Les anguilles. Payot, Paris, 218 p.

BRUSLÉ J., 1989. Les migrations au cours du cycle biologique des anguilles. Oceanis, 15 , 181-195.

CHISNALL B.L., HAYES J.W., 1991. Age and growth of shortfinned eels (Anguilla australis) in the lower Waikato basin, North Island, New Zealand. N.Z. J. Mar. Freshw. Res., $25,71-80$.

DEELDER C.L., 1957. On the growth of eels in the IJsselmeer. J. Cons. Per. Inter. Expl. Mer, 23, 83-88.

DEELDER C.L., 1970. Synopsis of biological data on the eel Anguilla anguilla L., FAO fishery Synopsis $N^{\circ} 80$, Rome.

DEELDER C.L., 1981-82. On the age and growth of cultured eels, Anguilla anguilla (Linnaeus, 1758). Aquaculture, 26, 13-22.

DEKKER W., 1986. Age reading of European eels using tetracycline labelled otoliths. ICES Anad. Catad. Fish Comm. C.M. 1986/M :16: 14 p.

EGUSA S., 1979. Notes on the culture of the European eel (Anguilla anguilla L.) in Japanese farming ponds. Rapp. P.-v. Réun. Cons. int. Explor. Mer, 174, 51-58.

FERNÀNDEZ-DELGADO C., HERNANDO J.A., HERRERA M., BELLIDO M., 1989. Age and growth of yellow eels, Anguilla anguilla, in the estuary of the Guadalquivir river (South-West Spain). J. Fish Biol., 34, 561-570.

FONTAINE M., 1989. Les Anguilles : Migration et reproduction. Oceanis, 15, 197-206.

FONTAINE M., 1992. Les Anguilles. Le rôle joué par l'Institut océanographique, Fondation Albert $1^{\text {er }}$ Prince de Monaco, dans le développement de nos connaissances sur leur biologie. Bull. Inst. océanog., Monaco, $N^{\circ}$ spécial 10, 5-16.

FONTENELLE G., 1991. Age et longueur moyenne des anguilles (Anguilla anguilla) en Europe: une revue critique. EIFAC Working Party on Eel, Dublin, Ireland. $16 \mathrm{p}$.

GORDO L.S., JORGE M.I., 1991. Age and growth of the European eel, Anguilla anguilla (Linnaeus, 1758) in the Aveiro lagoon, Portugal. Sci. Mar., 55, 389-395.

HANSEN R.A., EVERSOLE A.G., 1984. Age, growth, and sex ratio of American eels in brackish-water portions of a South Carolina river. Trans. Am. Fish. Soc., 113, 744-749.

KUHLMANN H., 1979. The influence of temperature, food, initial size, and origin of elvers (Anguilla anguilla L.). Rapp. P.-v. Réun. Cons. int. Explor. Mer, 174, 59-63.

LECOMTE-FINIGER R., 1985. L'âge de l'anguille européenne (Anguilla anguilla L., 1758) : état actuel des connaissances et recherches nouvelles en Méditerranée. $Z$. angew. Ichthyol., 4, 178-192.

LECOMTE-FINIGER R., 1990. Métamorphose de l'anguille jaune en anguille argentée Anguilla anguilla L. et sa migration catadrome. Ann. Biol., 29, 183-194.

LECOMTE-FINIGER R., 1992. Growth history and age at recruitment of European glass eels (Anguilla anguilla) as revealed by otolith microstructure. Mar. Biol., 114, 205-210.

LECOMTE-FINIGER R., YAHYAOUI A. 1989. La microstructure de l'otolithe au service de la connaissance du développement larvaire de l'Anguille européenne Anguilla anguilla. C.R. Acad. Sci., 308 (série III), 1-7.

LEE T.-W., LASSERRE G., 1979. Analyse de la structure et estimation du stock d'une population d'anguilles d'un réservoir à poissons du bassin d'Arcachon. Bull. Ecol., $10,139-145$. 
MCEWAN A., HECHT T., 1984. Age and growth of the longfin eel, Anguilla mossambica Peters, 1852 (Pisces : Anguillidae) in Transkei rivers. S. Afr. J. Zool., 19, 280-285.

MEUNIER F.J., 1994. Etude des populations d'anguilles du Rhin. Quelques résultats expérimentaux. In WEIDMANN H., MEDER H., Sandoz-Rheinfonds, Compte $r$ endu du Symposium du 3/4 septembre 1992 , 134-137, Sandoz A.G., Bâle.

MICHAUD M., DUTIL J.-D., DODSON J.J., 1988. Determination of the age of young American eels, Anguilla rostrata, in fresh water, based on otolith surface area and microstructure. J. Fish Biol., 32, 179-189.

MORIARTY C., 1983. Age determination and growth rate of eels, Anguilla anguilla (L.). J. Fish Biol., 23, 257-264.

MOUNAIX B., 1992. Intercalibration et validation des méthodes d'estimation de l'âge de l'Anguille européenne (Anguilla anguilla, L.). Application au bassin versant de la Vilaine, Bretagne. Thèse de Doctorat d'Halieutique, ENSA Rennes, France, $146 \mathrm{p}$.

NAGIEC M., BAHNSAWY M.H., 1990. Age and growth of female eels, Anguilla anguilla L., in a Polish lake, Jeziorak lake, Mazurian lake district, Poland. Aquacult. Fish. Manage., 21, 459-470.

NIELSEN J., 1988. A review on marking and tagging methods applied to eel (Anguilla anguilla (L.)). FAO EIFAC Occas. Pap., 21, 24 p.

PANFILI J., 1993. Estimation de l'âge individuel des poissons : méthodologies et applications à des populations naturelles tropicales et tempérées. Thèse doctorat, Univ. Montpellier II Sci. Tech. Languedoc, 1992, Travaux et Documents, ORSTOM, Paris, $\mathrm{N}^{\circ} 112,456 \mathrm{p}$.

PANFILI J., XIMÉNES M.C., CRIVELLI A.J., 1994. Sources of variation in growth of the European eel (Anguilla anguilla) estimated from otoliths. Can. J. Fish. Aquat. Sci., 51, 506-515.

PAULOVITS G., BIRÕ P., 1986. Age determination and growth of eel, Anguilla anguilla (L.) in Lake Ferto, Hungary. Fish. Res., 4, 101-110.

POOLE R., 1991. The age and growth of eels (Anguilla anguilla L.) in two oligotrophic streams in the west of Ireland. EIFAC Working Party on Eel, Dublin, Ireland, 12 p.

RASMUSSEN G., THERKILDSEN B., 1979. Food, growth, and production of Anguilla anguilla L. in a small Danish stream. Rapp. P.-v. Réun. Cons. int. Explor. Mer, 174, 32-40.

ROSSI R., VILLANI P., 1980. A biological analysis of eel catches, Anguilla anguilla L., from the lagoons of Lesina and Varano, Italy. J. Fish Biol., 16, 413-423.

RUIZHANG GUAN, WUEHONG WANG, GUOZHEN KE, 1994. Age and growth of eels Anguilla japonica in a Chinese river. J. Fish Biol., 45, 653-660.

SINHA V.R.P., JONES J.W., 1967. On the age and growth of freshwater eel (Anguilla anguilla) . J. Zool., Lond., 153, 99-117.

TESCH F.W., 1977. The eel. Biology and management of anguillid eels. Chapman and Hall, London, $435 \mathrm{p}$.

TZENG W.N., TSAI Y.C., 1992. Otolith microstructure and daily age of Anguilla japonica, Temminck \& Schlegel elvers from the estuaries of Taiwan with reference to unit stock and larval migration. J. Fish Biol., 40, 845-857.

TZENG W.N., TSAI Y.C., 1994. Changes in otolith microchemistry of the Japanese eel, Anguilla japonica, during its migration from the ocean to the rivers of Taiwan. J. Fish Biol., 45, 671-683.

VØLLESTAD L.A., 1985. Age determination and growth of yellow eels, Anguilla anguilla (L.), from a brackish water, Norway. J. Fish Biol., 26, 521 -525.

VØLLESTAD L.A., 1986. Growth and production of female yellow eels (Anguilla anguilla L.) from brackish water in Norway. Vie Milieu, 36, 267-271. 
Bull. Fr. Pêche Piscic. (1994) 335 : 133-144 - 144-

VØLLESTAD L.A., 1989. Effect of growth rate on age at maturity of the European eel Anguilla anguilla. EIFAC Working Party on Eel, Porto, Portugal, $22 \mathrm{p}$.

VØLLESTAD L.A., 1992. The geographic variation in age and length at metamorphosis of maturing European eel : environmental effects and phenotypic plasticity. J. Anim. Ecol., 61, 41-48.

VØLLESTAD L.A., JONSSON B., 1988. A 13-year study of the population dynamics and growth of the European eel Anguilla anguilla in a Norwegian river : evidence for density-dependent mortality, and development of a model for predicting yield. J. Anim. Ecol., 57, 983-997.

VØLLESTAD L.A., LECOMTE-FINIGER R., STEINMETZ B., 1988. Age determination of Anguilla anguilla and related species. EIFAC Occas. Pap., 21, $28 \mathrm{p}$.

WICKINS J.F., 1985. Effects of size, culling and social history on growth of cultured elvers, Anguilla anguilla (L.). J. Fish Biol., 31, 71-82. 